\title{
Buenas prácticas en propiedad intelectual y MOOC: una experiencia Good practices in intellectual property and MOOC: an experience
}

\author{
Mª Clara Ubieto-Artur ${ }^{1}$, Lola Hernández Ara², $\mathbf{M}^{\mathrm{a}}$. Isabel Ubieto-Artur ${ }^{3}$, Pedro José Bueso Guillén ${ }^{4}$, María Luisa \\ Sein-Echaluce Lacleta ${ }^{5}$, Ángel Fidalgo Blanco ${ }^{6}$, Concepción Bueno García ${ }^{7}$ \\ cubieto@unizar.es, lara@unizar.es, iubieto@unizar.es, pbueso@unizar.es,mlsein@unizar.es, angel.fidalgo@upm.es, \\ cbueno@unizar.es
}

\author{
${ }^{1}$ Departamento de Filología Inglesa y Alemana \\ Universidad de Zaragoza \\ Zaragoza, España \\ ${ }^{3}$ Departamento de Ciencias de la Documentación \\ Universidad de Zaragoza \\ Zaragoza, España
}

${ }^{5}$ Departamento de Matemática Aplicada Universidad de Zaragoza

Zaragoza, España

\author{
${ }^{2}$ Biblioteca de la Facultad de Derecho \\ Universidad de Zaragoza \\ Zaragoza, España
}

${ }^{4}$ Departamento de Derecho de la Empresa Universidad de Zaragoza Zaragoza, España

${ }^{6}$ Departamento de Ingeniería Geológica y Minera Universidad Politécnica de Madrid Madrid, España

\footnotetext{
${ }^{7}$ Departamento de Ciencias de la Educación Universidad de Zaragoza

Zaragoza, España
}

\begin{abstract}
Resumen- Existe una creciente necesidad entre las instituciones educativas de que sus miembros estén informados y formados en las normas éticas y legales que rigen el uso de obras propias y ajenas. Es necesario sensibilizar a la comunidad educativa sobre el respeto a los derechos de autor y difundir buenas prácticas para el uso de la propiedad intelectual. Con esos objetivos se ha creado un curso masivo abierto en línea (MOOC) titulado "Buenas Prácticas en el Uso Académico de la Propiedad Intelectual”, que se ha llevado a cabo entre abril y mayo de 2017. El curso presenta y resuelve situaciones concretas con las que se enfrentan diariamente el profesorado y el alumnado en la creación de materiales didácticos y la realización de actividades académicas. Se presenta el análisis de los resultados de la implementación de este MOOC en cuanto a la consecución de sus objetivos y la satisfacción.
\end{abstract}

\section{Palabras clave: MOOC, Propiedad intelectual, Educación}

\begin{abstract}
The members of the educational institutions need to be informed and trained in the ethical and legal norms that govern the use of their own and of other authors. It is necessary to sensitize educational communities on the respect for copyright and to disseminate among them good practices for the use of intellectual property. With these goals, an innovation group from the University of Zaragoza (Spain) has created a massive open online course (MOOC) entitled "Good Practices in the Academic Use of Intellectual Property", which took place between April and May 2017. The course presents and resolves concrete situations which teachers and students face daily in the creation of didactic materials or during the realization of academic activities. The article analysis the results of the implementation of the MOOC, the achievement of their goals and the satisfaction of the participants.
\end{abstract}

Keywords: MOOC, Intellectual Property, Education

\section{INTRODUCCIÓN}

Entre las actividades propias del profesorado y el alumnado, la elaboración de trabajos académicos de diversa índole (trabajos de curso, artículos, proyectos, presentaciones, Trabajos Fin de Titulación,...) ha cobrado una mayor relevancia si cabe desde que el aprendizaje del estudiante se ha situado en el centro del proceso de enseñanza-aprendizaje.

Estos trabajos académicos requieren de un proceso de búsqueda, selección, recopilación, cita y referencia de aquellos materiales que sirven de base para su realización.

Por otro lado, la proliferación en la solicitud de estos trabajos y el fácil acceso a diverso material digital, favorecen el "corta y pega" prescindiendo de planteamientos éticos y/o legales.

Precisamente, autores como Area y Guarro (2012) afirman que las iniciativas de diversos organismos para promover la alfabetización informacional se centran en la enseñanza de procedimientos de búsqueda de información en bases de datos bibliográficas, pero suelen obviar el desarrollo de las competencias para generar y comunicar información. Es decir, las capacidades que nos permiten, una vez localizadas y seleccionadas las fuentes bibliográficas sobre una temática, generar un nuevo conocimiento y difundirlo adecuadamente.

En este sentido, percibimos que la comunidad educativa necesita sensibilización, información y formación sobre la adecuación de la ley de propiedad intelectual y los derechos de autor para el correcto desarrollo de las actividades 
especialmente relacionadas con la generación de nuevo conocimiento y su difusión.

La adquisición de estas capacidades es necesaria también para estudiantes y docentes de niveles no universitarios y es posible conseguirla en contextos de aprendizaje diversos (Area y Guarro, 2012). La participación en un curso en línea puede ser uno de ellos.

Para cubrir esta necesidad se ha diseñado e implementado un MOOC (Massive Open Online Course) en MiriadaX, dedicado a las "Buenas prácticas en el uso académico de la propiedad intelectual”, puesto que este tipo de cursos son adecuados para la difusión del conocimiento, la innovación pedagógica y la sensibilización (Teixeira, García-Cabot, García-López, Mota, y De-Marcos, 2015) y además constituyen una apuesta estratégica de la Universidad de Zaragoza.

En la primera edición del MOOC se han inscrito 2.915 de los que iniciaron el curso 1.369 y lo finalizaron 758. Han revisado cuestiones relativas a buenas prácticas en materia de propiedad intelectual desde cuatro ejes: el autor como generador de conocimiento, como gestor de conocimiento ajeno, como docente y desde la perspectiva legal.

\section{CONTEXTO}

Es posible encontrar bibliografía muy diversa relacionada con el tema de la propiedad intelectual y la educación, tanto universitaria como preuniversitaria, pero hasta la fecha no se ha publicado ningún MOOC sobre esta temática.

El público al que va dirigido el MOOC es el alumnado y profesorado universitario de cualquier curso y titulación, aunque también puede resultar interesante para estos mismos colectivos de otros niveles educativos. Se recomienda preferentemente a personas no iniciadas en el tema y durante el primer año de carrera, aunque puede ser útil para cualquier persona que desee realizar trabajos académicos.

La duración del curso ha sido de cinco semanas, cuarenta horas de estudio estimadas, adecuada al perfil del participante esperado.

El objetivo general de la comunicación es presentar los resultados y el análisis de los datos obtenidos en la implementación del MOOC, siendo los objetivos específicos los siguientes:

- Estudio del grado de satisfacción de los participantes en el MOOC.

- Estudio del impacto del MOOC en la comunidad universitaria de la UZ.

- Elaboración de 'lecciones aprendidas' a partir de las aportaciones de los participantes para mejorar futuras ediciones del MOOC.

- Mejora y puesta al día (si es necesario) del material audiovisual del MOOC.

\section{DESCRIPCIÓN}

En primer lugar, y teniendo presente que el curso puede ser realizado por cualquier persona, se estableció el perfil al que el curso estaba dirigido preferentemente. En nuestro caso, profesorado de cualquier nivel y estudiantes a partir de 12 años. Esta decisión permitió un mejor ajuste del curso a las necesidades e intereses de los posibles participantes.

Así, los contenidos, las actividades, los ejemplos, los recursos o el material complementario presentan situaciones concretas que surgen a la hora de elaborar trabajos académicos o de investigación.

Todos los MOOC de la plataforma MiriadaX se inician con un breve vídeo de presentación del curso así como con una sucinta descripción del mismo, en los que se resumen los objetivos y se presentan brevemente los contenidos del mismo. A continuación se accede a los módulos del MOOC.

La estructura del curso es modular, para facilitar el seguimiento adaptado a los intereses de los participantes puesto que era posible acceder indistintamente a cualquier módulo desde la primera del curso. El curso consta de cinco módulos, el primero es de presentación del mismo e incluye la encuesta inicial, que ha de ser cumplimentada obligatoriamente por los participantes.

La encuesta inicial consta de 10 preguntas y tiene por objetivo obtener información básica sobre el perfil de los participantes como: edad, género, nivel de estudios, perfil profesional actual, etc. Incluye también preguntas sobre conocimientos previos de los participantes sobre el tema.

Cada módulo cuenta con una breve presentación por medio de un vídeo y su correspondiente transcripción de voz a texto, en el que se resumen los objetivos del módulo por medio de algunas de las preguntas que van a ser contestadas a lo largo del mismo. Este diseño obedece al hecho de que cada participante pueda elegir tanto los módulos a realizar como la secuenciación de los mismos de acuerdo con sus intereses y necesidades. Las preguntas fomentan el seguimiento del curso al presentar retos y desafíos a los participantes. Algún ejemplo de dichas preguntas son:

- ¿De qué hablamos cuando nos referimos a los derechos de autor? (Módulo 1)

- ¿El hecho de citar significa que no hay plagio? (Módulo 1)

- ¿Cómo se protegen los derechos de explotación de una obra? (Módulo 2).

- ¿Cómo se protegen los derechos de explotación de una obra? (Módulo 2).

- ¿Qué es una licencia Creative Commons? (Módulo 2).

- ¿QQué es al acceso abierto? (Módulo 2).

- ¿¿Puedo publicar en abierto? (Módulo 2).

- ¿Qué se considera una mala práctica en cuanto a la propiedad intelectual? (Módulo 3).

- ¿Cómo evitar y prevenir las malas prácticas en el aula? (Módulo 3). 
Cada módulo se despliega en un número variable de apartados. Los primeros incluyen siempre el material para el estudio, y los dos últimos se dedican al material complementario y al cuestionario obligatorio.

El material para el estudio incluye un vídeo didáctico de entre cinco y diez minutos de duración, la transcripción del audio y la copia de la presentación, ambas en formato pdf. Este material se articula en torno a diversas preguntas a las que se da respuesta según se avanza en el desarrollo del material.

El material complementario, en formato html, va dirigido para quienes deseen ampliar sus conocimientos sobre el tema o conocer distintos recursos. Se presenta mediante referencias a artículos científicos, recursos educativos abiertos (OER: open educational resources), aplicaciones web gratuitas, vídeos, portales (bibliotecas, organismos europeos, etc.).

El cuestionario obligatorio de cada uno de los módulos permite comprobar el grado de conocimiento adquirido, puesto que las respuestas a algunas de las preguntas se encuentran literalmente en el material para el estudio, mientras que otras requieren la aplicación de lo aprendido a un caso o situación concretos. Se trata de un test de diez preguntas de opción múltiple, con tres posibles respuestas, de las que sólo una es correcta. ¿Qué derecho de explotación se ejerce cuando vemos una película en una sala de cine? es un ejemplo de pregunta que se contesta en el material. Un ejemplo de pregunta de aplicación es la que facilita una referencia bibliográfica y se pide que se identifique si es una cita, una referencia o un fragmento. Otro ejemplo sería la creación automática de una referencia mediante una aplicación abierta y en línea y la comparación del resultado obtenido con las opciones de respuesta a la pregunta.

Para superar el cuestionario era necesario responder correctamente al menos a cinco de las diez preguntas. Cada participante disponía de tres intentos para contestar las preguntas de cada cuestionario. Al enviar el cuestionario cumplimentado, el participante obtenía feedback de cada una de sus respuestas, tanto de las correctas como de las incorrectas. En este último caso, se les dirigía hacia el apartado del material de estudio que debía revisar para responder correctamente. Para obtener el certificado del curso era necesario contestar correctamente a los cuatro cuestionarios.

Para facilitar la interacción entre los participantes del MOOC se ha contado con un foro general y un foro para cada uno de los módulos. El foro general se destinaba a cuestiones relativas al funcionamiento general del curso. Por ejemplo se enviaron los mensajes de bienvenida, de finalización o de apoyo. Los participantes inscritos se presentaron o avisaron de diversos problemas técnicos. Se ha intentado que los mensajes enviados a los foros se centraran en la temática del módulo para que así fuese más fácil el seguimiento por parte del profesorado y de los inscritos interesados en el mismo. No siempre se ha conseguido porque los participantes o bien plantean dudas antes de revisar el material de otros módulos, anticipando sus dudas, o bien no siguen el hilo adecuado para enviar mensajes al foro.
El último módulo contiene además la encuesta final del curso que es obligatoria. Consta de doce preguntas, algunas de ellas encaminadas a obtener información básica como el género, la edad, la nacionalidad, el nivel de estudios o el perfil profesional actual. Otras dedicadas a conocer los módulos finalizados antes de cumplimentar la encuesta final, la valoración sobre la utilidad de los contenidos y sobre lo aprendido en el curso. Y, para finalizar, tres preguntas abiertas para indicar los tres aspectos que más les han gustado, los que menos y los que podrían mejorarse.

\section{Resultados}

El número total de participantes que se inscribieron en el MOOC ascendió a 2.915. De ellos, participó en el curso el 46,96\% (1.369). Si se tiene en cuenta el número de personas que finalizaron el curso, 758, encontramos que la tasa de abandono es del 5,3\% y una tasa de finalización es de 55,56\%, considerando los estudiantes activos, que son los que al menos comenzaron con la encuesta inicial. Estos resultados están muy por encima de la media habitual en este tipo de cursos que es del 9,8\% (Leris, Sein-Echaluce, Hernández y FidalgoBlanco, 2016).

Por módulos, el porcentaje de personas que los finalizaron se muestra en la tabla 1 :

Tabla 1: $\mathbf{N}^{\circ}$. de participantes por módulo

\begin{tabular}{|l|c|c|c|}
\hline \multicolumn{1}{|c|}{ Módulos } & $\begin{array}{c}\text { Norsonas } \\
\text { que } \\
\text { iniciaron el } \\
\text { módulo }\end{array}$ & $\begin{array}{c}\mathbf{N}^{\mathbf{0}} \mathbf{d e} \\
\text { personas } \\
\text { que } \\
\text { finalizaron } \\
\text { el módulo }\end{array}$ & $\begin{array}{c}\text { Tasa de } \\
\text { finalización }\end{array}$ \\
\hline $\begin{array}{l}\text { Módulo 0. } \\
\text { Presentación }\end{array}$ & 1274 & 1242 & 97,5 \\
\hline $\begin{array}{l}\text { Módulo 1. } \\
\text { La creación } \\
\text { de un trabajo } \\
\text { académico 0 } \\
\text { científico }\end{array}$ & 1260 & 968 & 76,8 \\
\hline $\begin{array}{l}\text { Módulo 2. } \\
\text { Propiedad } \\
\text { Intelectual } \\
\text { Básica }\end{array}$ & 1003 & 868 & 86,5 \\
\hline $\begin{array}{l}\text { Módulo 3. } \\
\text { Propiedad } \\
\text { Intelectual y } \\
\text { Docencia: } \\
\text { buenas } \\
\text { prácticas }\end{array}$ & 905 & 827 & 91,4 \\
\hline $\begin{array}{l}\text { Módulo 4. } \\
\text { Perspectiva } \\
\text { Legal de la } \\
\text { Propiedad } \\
\text { Intelectual }\end{array}$ & 866 & 769 & \\
\hline & & & \\
\hline
\end{tabular}


La encuesta inicial fue cumplimentada por 1.233 participantes y la encuesta final por 775. Señalamos a continuación algunos de los resultados obtenidos:

- El 50\% de los que manifestaron su satisfacción general con el curso, se mostraron muy satisfechos y el $44 \%$ bastante satisfechos.

- Al menos $10 \%$ de los que realizan la encuesta final estaban vinculados a la Universidad de Zaragoza.

- Un alto porcentaje de participantes (84,65\%) que llevan a cabo la encuesta final cumplimentan la pregunta número 9 (Indica los 3 aspectos que MÁs te han gustado del curso).

- Algo menos de la mitad (46,71\%) de los participantes que llevan a cabo la encuesta final cumplimentan la pregunta número 10 (Indica los 3 aspectos que MENOS te han gustado del curso).

- Aproximadamente la mitad de los participantes (49,42\%) que llevan a cabo la encuesta final cumplimentan la pregunta número 11 (Indica los aspectos que crees que podrían MEJORAR el curso).

- Número total de ítems recogidos en las respuestas a estas tres preguntas: 2.634 .

Las respuestas más frecuentes a la pregunta sobre los aspectos que más han gustado del curso -en cuanto a los contenidos- son aquellas que los califican de ilustrativo (32,5\%). El aspecto que se cita más frecuentemente entre los menos valorados se refiere a la necesidad de ampliación y de mayor profundidad en algunos contenidos (28\%). En coherencia con estos resultados, la sugerencia de mejora más frecuencia ha sido precisamente que en el curso se ampliaran ciertos contenidos.

\section{CONCLUSIONES}

El MOOC va a tener una segunda edición, puesto que se ha constatado el interés y la necesidad a partir del número de participantes interesados y retroalimentación que los inscritos han ofrecido a través de la encuesta final y de su participación en los foros.

El trabajo en equipo del grupo de ponentes ha permitido llevar adelante el MOOC de manera que se han contestado dudas de una manera rápida y eficaz y ha sido posible compaginar la atención a los inscritos con sus tareas habituales.

Los participantes han manifestado en general una alta satisfacción con el curso. El hecho de que haya considerablemente más respuestas que resaltan aspectos positivos del mismo en detrimento de los negativos es también un indicador de esa satisfacción.

Como curso masivo abierto y en línea es totalmente transferible en cuanto a su diseño e implementación.

Entre las recomendaciones para futuras ediciones podemos destacar los siguientes aspectos, que se deben mantener o potenciar:

- Claridad expositiva.

- La oferta de material adicional complementario y por escrito.

- El carácter ilustrativo e informativo del curso.

- Su orientación práctica.

Entre los aspectos que se deberían revisarse se encuentran:

- $\quad$ La calidad técnica de los vídeos.

- Y la advertencia de que la propiedad intelectual se aborda desde la normativa española.

\section{AGRADECIMIENTOS}

Nuestro agradecimiento a la Universidad de Zaragoza por su apoyo en la realización del Proyecto de Innovación Docente PIIDUZ_16_421.

\section{REFERENCIAS}

Area, M., y Guarro, A. (2012). La alfabetización informacional y digital: fundamentos pedagógicos para la enseñanza y el aprendizaje competente. Revista española de Documentación Científica, 35 (Monográfico), 46-74. Doi: http://dx.doi.org.roble.unizar.es:9090/10.3989/redc.2012 .v35.imonografico

Leris López, D., Sein-Echaluce Lacleta, M. L., , Hernández, M., and Fidalgo-Blanco, Á. (2016). Participantes heterogéneos en MOOCs y sus necesidades de aprendizaje adaptativo. Education in the Knowledge Society (EKS), 17(4), 91-109. DOI=https://doi.org/10.14201/eks201617491109

Teixeira, A., Garcia-Cabot, A., Garcia-Lopez, E., Mota, J., \& de-Marcos, L. (2015). A New Competence-based Approach for Personalizing MOOCs in a Mobile Collaborative and Networked Environment. RIED. Revista Iberoamericana de Educación a Distancia, 19(1),

143-160. 